\title{
Front Matter: Volume 7231
}

, "Front Matter: Volume 7231," Proc. SPIE 7231, Light-Emitting Diodes: Materials, Devices, and Applications for Solid State Lighting XIII, 723101 (23 February 2009); doi: 10.1117/12.823976

EPent: SPIE OPTO: Integrated Optoelectronic Devices, 2009, San Jose, SPIE. California, United States 


\title{
PROCEEDINGS OF SPIE
}

\section{Light-Emitting Diodes: Materials, Devices, and Applications for Solid State Lighting XIII}

\author{
Klaus P. Streubel \\ Heonsu Jeon \\ Li-Wei Tu \\ Editors
}

27-29 January 2009

San Jose, California, United States

Sponsored and Published by

SPIE

Cosponsored by

AIXTRON AG (Germany)

OSRAM Opto Semiconductors GmbH (Germany) 
The papers included in this volume were part of the technical conference cited on the cover and title page. Papers were selected and subject to review by the editors and conference program committee. Some conference presentations may not be available for publication. The papers published in these proceedings reflect the work and thoughts of the authors and are published herein as submitted. The publisher is not responsible for the validity of the information or for any outcomes resulting from reliance thereon.

Please use the following format to cite material from this book:

Author(s), "Title of Paper," in Light-Emitting Diodes: Materials, Devices, and Applications for Solid State Lighting XIII, edited by Klaus P. Streubel, Heonsu Jeon, Li-Wei Tu, Proceedings of SPIE Vol. 7231 (SPIE, Bellingham, WA, 2009) Article CID Number.

ISSN 0277-786X

ISBN 9780819474773

Published by

SPIE

P.O. Box 10, Bellingham, Washington 98227-0010 USA

Telephone +1 3606763290 (Pacific Time) · Fax +1 3606471445

SPIE.org

Copyright (c) 2009, Society of Photo-Optical Instrumentation Engineers

Copying of material in this book for internal or personal use, or for the internal or personal use of specific clients, beyond the fair use provisions granted by the U.S. Copyright Law is authorized by SPIE subject to payment of copying fees. The Transactional Reporting Service base fee for this volume is $\$ 18.00$ per article (or portion thereof), which should be paid directly to the Copyright Clearance Center (CCC), 222 Rosewood Drive, Danvers, MA 01923. Payment may also be made electronically through CCC Online at copyright.com. Other copying for republication, resale, advertising or promotion, or any form of systematic or multiple reproduction of any material in this book is prohibited except with permission in writing from the publisher. The CCC fee code is 0277-786X/09/\$18.00.

Printed in the United States of America.

Publication of record for individual papers is online in the SPIE Digital Library.

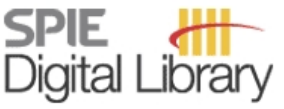

SPIEDigitalLibrary.org

Paper Numbering: Proceedings of SPIE follow an e-First publication model, with papers published first online and then in print and on CD-ROM. Papers are published as they are submitted and meet publication criteria. A unique, consistent, permanent citation identifier (CID) number is assigned to each article at the time of the first publication. Utilization of CIDs allows articles to be fully citable as soon they are published online, and connects the same identifier to all online, print, and electronic versions of the publication. SPIE uses a six-digit CID article numbering system in which:

- The first four digits correspond to the SPIE volume number.

- The last two digits indicate publication order within the volume using a Base 36 numbering system employing both numerals and letters. These two-number sets start with 00, 01, 02, 03, 04 , 05, 06, 07, 08, 09, OA, OB ... 0Z, followed by 10-1Z, 20-2Z, etc.

The CID number appears on each page of the manuscript. The complete citation is used on the first page, and an abbreviated version on subsequent pages. Numbers in the index correspond to the last two digits of the six-digit CID number. 


\section{Contents}

vii Conference Committee

\section{LED DESIGN AND FABRICATION I}

7231 OA Color-consistent LED modules for general lighting (Invited Paper) [7231-09]

C. Hoelen, P. van der Burgt, Philips Lighting (Netherlands); P. Jungwirth, Philips Lighting

(Canada); M. Keuper, Philips Lighting (Netherlands); K. Man, Philips Lighting (Canada);

C. Mutter, J.-W. ter Weeme, Philips Lighting (Netherlands)

7231 OC Beam-shaping properties of InGaN thin-film micro-cavity light-emitting diodes with photonic crystals [7231-11]

K. Bergenek, OSRAM Opto Semiconductors GmbH (Germany) and Univ. of St. Andrews (United Kingdom); Ch. Wiesmann, H. Zull, C. Rumbolz, R. Wirth, N. Linder, K. Streubel, OSRAM Opto Semiconductors GmbH (Germany); T. F. Krauss, Univ. of St. Andrews (United Kingdom)

7231 OE Growth of staggered InGaN quantum well light-emitting diodes emitting at $\mathbf{5 2 0 - 5 2 5 ~} \mathbf{n m}$ employing graded temperature profile [7231-13]

H. Zhao, G. Liu, X. Li, R. A. Arif, G. S. Huang, Y.-K. Ee, N. Tansu, Lehigh Univ. (United States)

UV LEDs

$72310 G \quad$ MOVPE growth for UV-LEDs (Invited Paper) [7231-15]

A. Knaver, F. Brunner, Ferdinand-Braun-Institut für Höchstfrequenztechnik (Germany);

T. Kolbe, Technische Univ. Berlin (Germany); V. Küller, H. Rodriguez, S. Einfeldt, M. Weyers, Ferdinand-Braun-Institut für Höchstfrequenztechnik (Germany); M. Kneissl, Ferdinand-BraunInstitut für Höchstfrequenztechnik (Germany) and Technische Univ. Berlin (Germany)

7231 OJ Effect of UV irradiation on the apoptosis and necrosis of Jurkat cells using UV LEDs [7231-18] S. A. Inada, H. Amano, I. Akasaki, Meijo Univ. (Japan); A. Morita, K. Kobayashi, Nagoya City Univ. (Japan)

7231 OK Reduced non-thermal roll-over in violet-emitting GalnN wide-well LEDs grown on low-dislocation-density substrates [7231-56]

M. Maier, T. Passow, M. Kunzer, W. Schirmacher, W. Pletschen, L. Kirste, K. Köhler, J. Wagner, Fraunhofer-Institut für Angewandte Festkörperphysik (Germany)

LED MANUFACTURING

723100 Decay of lumen and chromaticity of high-power phosphor-converted white-light-emitting diodes in thermal aging (Invited Paper) [7231-23]

J.-S. Wang, C.-C. Tsai, M.-H. Chen, National Sun Yat-Sen Univ (Taiwan); Y.-C. Hsu, National Pingtung Univ. of Science and Technology (Taiwan); H.-L. Hu, C.-W. Lee, ITRI (Taiwan);

W.-H. Cheng, National Sun Yat-Sen Univ. (Taiwan) 
7231 OP Photoluminescence mapping as a tool to improve LED production (Invited Paper) [7231-24] Z. Li, T. Ryan, Nanometrics Inc. (United States)

$72310 Q \quad$ High-performance heat sink for solid-state lighting [7231-25]

J. Vetrovec, A. Litt, Aqwest LLC (United States)

LED DESIGN AND FABRICATION II

7231 OU Enhancement of light extraction efficiency of InGaN quantum well light-emitting diodes with polydimethylsiloxane concave microstructures [7231-30]

Y.-K. Ee, P. Kumnorkaew, H. Tong, R. A. Arif, J. F. Gilchrist, N. Tansu, Lehigh Univ. (United States)

7231 OV A 4.26- $\mu \mathrm{m}$ RCLED and a fast low-power $\mathrm{CO}_{2}$ sensor [7231-31]

A. Nelson, A. Kouznetsov, GE Sensing (United States)

7231 OW Electrical and optical characteristics of green light-emitting diodes grown on bulk GaN substrates [7231-32]

Y. Yang, X. A. Cao, C. H. Yan, West Virginia Univ. (United States)

\section{PHOSPHORS}

7231 OX Up and down: color conversion for solid-state lighting (Invited Paper) [7231-33]

P. Hartmann, P. Pachler, Ledon Lighting Jennersdorf GmbH (Austria); E. L. Payrer, TU Graz (Austria); S. Tasch, Ledon Lighting Jennersdorf GmbH (Austria)

723111 Improved predictive modeling of white LEDs with accurate luminescence simulation and practical inputs with TracePro opto-mechanical design software [7231-37]

C. Tsao, E. R. Freniere, Lambda Research Corp. (United States); L. Smith, Ceres (United States)

\section{NANOSTRUCTURES}

723112 Growth and luminescence properties of one-dimensional InN and InGaN nanostructures (Invited Paper) [7231-38]

L.-C. Chen, A. Ganguly, National Taiwan Univ. (Taiwan); C.-W. Hsu, M.-S. Hu, Institute of Atomic and Molecular Sciences (Taiwan); S.-P. Fu, Y.-F. Chen, National Taiwan Univ. (Taiwan); K.-H. Chen, National Taiwan Univ. (Taiwan) and Institute of Atomic and Molecular Sciences (Taiwan) 
723116 High-quality free-standing GaN thick-films prepared by hydride vapor phase epitaxy using stress reducing techniques (Invited Paper) [7231-42]

H.-H. Huang, W.-I. Lee, National Chiao Tung Univ. (Taiwan); K.-M. Chen, National Chiao Tung Univ. (Taiwan) and Industrial Technology Research Institute (Taiwan); T.-L. Chu, P.-L. Wu, H.-W. Yu, National Chiao Tung Univ. (Taiwan); P.-C. Liu, C.-L. Chao, T.-W. Chi, J.-D. Tsay, Industrial Technology Research Institute (Taiwan); L.-W. Tu, National Sun Yat-Sen Univ. (Taiwan)

723118 GaN-based LEDs grown on 6-inch diameter Si (111) substrates by MOVPE (Invited Paper) [7231-44]

D. Zhu, C. McAleese, K. K. McLaughlin, M. Häberlen, C. O. Salcianu, E. J. Thrush, M. J. Kappers, W. A. Phillips, Univ. of Cambridge (United Kingdom); P. Lane, D. J. Wallis, T. Martin, M. Astles, QinetiQ (United Kingdom); S. Thomas, A. Pakes, M. Heuken, Aixtron, Ltd. (United Kingdom); C. J. Humphreys, Univ. of Cambridge (United Kingdom)

723119 Development of new substrate technologies for GaN LEDs: atomic layer deposition transition layers on silicon and ZnO (Invited Paper) [7231-45]

W. E. Fenwick, N. Li, M. Jamil, T. Xu, A. Melton, S. Wang, H. Yu, Georgia Institute of Technology (United States); A. Valencia, J. Nause, Cermet, Inc. (United States); C. Summers,

I. T. Ferguson, Georgia Institute of Technology (United States)

\section{POSTER SESSION}

7231 1E Stray light rejection techniques for LED measurements using CCD based spectrometers [7231-51]

J. Jablonski, A. Arecchi, J. Jacobs, T. Annicchiarco, SphereOptics, LLC (United States)

$7231 \mathrm{lF} \quad$ Simulator for assessing the performance of polychromatic LED light sources [7231-52] M. Bürmen, Univ. of Ljubljana (Slovenia); F. Pernuš, B. Likar, Univ. of Ljubljana (Slovenia) and Sensum Computer Vision Systems (Slovenia)

$7231 \mathrm{1H}$ Weak-microcavity organic light-emitting diodes with improved light-extraction and wide viewing-angle [7231-54]

S.-H. Cho, KAIST (Korea, Republic of) and Samsung SDI Co., Ltd. (Korea, Republic of); Y.-H. Lee, KAIST (Korea, Republic of); Y.-W. Song, Y.-C. Kim, J. Lee, J. H. Lee, K. H. Hwang, D.-S. Zang, Samsung SDI Co., Ltd. (Korea, Republic of) 
Downloaded From: https://www.spiedigitallibrary.org/conference-proceedings-of-spie on 25 Apr 2023

Terms of Use: https://www.spiedigitallibrary.org/terms-of-use 


\title{
Conference Committee
}

\author{
Symposium Chair \\ James G. Grote, Air Force Research Laboratory (United States) \\ Symposium Cochair
}

E. Fred Schubert, Rensselaer Polytechnic Institute (United States)

Program Track Chair

E. Fred Schubert, Rensselaer Polytechnic Institute (United States)

Conference Chairs

Klaus P. Streubel, OSRAM Opto Semiconductors GmbH (Germany)

Heonsu Jeon, Seoul National University (Korea, Republic of)

\section{Conference Cochair}

Li-Wei Tu, National Sun Yat-Sen University (Taiwan)

Program Committee

Gerd Bacher, Universität Duisburg-Essen (Germany)

Michael Heuken, AIXTRON AG (Germany)

Zhaoran Rena Huang, Rensselaer Polytechnic Institute (United States)

Satoshi Kamiyama, Meijo University (Japan)

Markus Klein, OSRAM Opto Semiconductors GmbH (Germany)

Michael R. Krames, Philips Lumileds Lighting Company (United States)

Kei May Lau, Hong Kong University of Science and Technology (Hong Kong China)

Kurt J. Linden, Spire Corporation (United States)

Hans Nikol, Philips Lighting B.V. (Netherlands)

E. Fred Schubert, Rensselaer Polytechnic Institute (United States)

Jerry A. Simmons, Sandia National Laboratories (United States)

Ross P. Stanley, Centre Suisse d'Electronique et de Microtechnique SA (Switzerland)

Session Chairs

1 LED Applications and SSL

Klaus P. Streubel, OSRAM Opto Semiconductors GmbH (Germany) 
LED Droop

E. Fred Schubert, Rensselaer Polytechnic Institute (United States)

3 LED Design and Fabrication I

Kei May Lau, Hong Kong University of Science and Technology (Hong Kong, China)

$4 \quad$ UV LEDS

Gerd Bacher, Universität Duisburg-Essen (Germany)

5 LED Manufacturing I

Michael Heuken, AIXTRON AG (Germany)

6 LED Manufacturing II

Ross P. Stanley, Centre Suisse d'Electronique et de Microtechnique SA (Switzerland)

7 LED Design and Fabrication II

Heonsu Jeon, Seoul National University (Korea, Republic of)

8 Phosphors

Hans Nikol, Philips Lighting B.V. (Netherlands)

9 Nanostructures

Satoshi Kamiyama, Meijo University (Japan)

10 Novel Substrates

Li-Wei Tu, National Sun Yat-Sen University (Taiwan)

11 Organic LEDs

Heonsu Jeon, Seoul National University (Korea, Republic of) 